\title{
Transmeatal Low Level Laser Therapy (LLLT) on Vestibular Inner Ear after Topical Gentamicin Ototoxicity
}

\author{
ChungKu Rhee ${ }^{1,2}$ \\ So-Young Chang ${ }^{2}$ \\ Phil-Sang Chung ${ }^{1,2}$ \\ Jin-Chul Ahn ${ }^{2,3}$ \\ Jae Yun Jung ${ }^{1,2}$
}

\footnotetext{
${ }^{1}$ Department of Otolaryngology-HNS, College of Medicine, Cheonan, Korea

${ }^{2}$ Medical Laser Research Center, Dankook University, Cheonan, Korea

${ }^{3}$ Biomedical Translational Research Institute, Dankook University, Cheonan, Korea
}

Received December 4, 2014

Revised December 11, 2014

Accepted December 12, 2014

\author{
Correspondence \\ ChungKu Rhee \\ Department of Otolaryngology-Head and Neck \\ Surgery, Dankook University College of Medicine, \\ 201, Manghyang-ro, Dongnam-gu, Cheonan 330- \\ 715 , Korea \\ Tel: +82-41-550-3975 \\ Fax: +82-41-556-1090 \\ E-mail: rheeck@dankook.ac.kr \\ (c) Korean Society for Laser Medicine and Surgery \\ (c) This is an open access article distributed under the \\ terms of the Creative Commons Attribution Non- \\ Commercial License (http://creativecommons.org/ \\ licenses/by-nc/3.0) which permits unrestricted non- \\ commercial use, distribution, and reproduction in any \\ medium, provided the original work is properly cited.
}

\begin{abstract}
Background and Objectives
The objectives of this study is to evaluate any adverse effects on outer and inner ear structures after transmeatal low level laser therapy (LLLT) and to see the effects of transmeatal LLLT on gentamicin induced unilateral vestibulopathy in guinea pigs.

\section{Materials and Methods}

We measured penetration rate of transmeatal low level laser (LLL) into the perilymphatic space of cochlea through a tympanic membrane, cochlea wall and hearing level post transmeatal LLLT were measured. Histopathologic studies on ear canal skin and ear drum were performed for observation of any damage by LLLT. Guinea pigs were then divided into control and LLLT group. Both groups received injection of gentamicin (GM) into the left middle ear. LLLT group were irradiated into the left ear through the external ear canal for 5 days starting 1 day after GM injection into the left ear. The animals underwent sinusoidal oscillation about a vertical and off vertical axis 5 days post GM injection.
\end{abstract}

\section{Results}

No damage on ear canal skin and ear drum was noted and hearing remained normal after LLLT. The penetration rate of LLL through the drum was $8 \%$ and $5 \%$ of LLL penetrated into the perilymphatic space. The left side gains of the control group were significantly decreased compared to the left side gain of the LLLT group. The gains of the right ear in both groups were not significantly different.

\section{Conclusion}

This study demonstrated that transmeatal LLLT restores vestibular function in guinea pigs with gentamicin induced ototoxic dysfunction. LLLT may have clinical implications in the treatment of various vestibular diseases with vestibular dysfunction.

\section{Key words}

LLLT; Gentamicin; Ototoxicity; SHA; Vestibular dysfunction 


\section{INTRODUCTION}

Low level laser therapy (LLLT) is the application of light in the range of 1-1000 $\mathrm{mW}$ to pathologic conditions to promote tissue regeneration, reduce inflammation, and relieve pain. The LLLT uses typically of narrow spectral width, in the red or near infrared (NIR) spectrum $1600-1000$ $\mathrm{nm}$ ), with a power density (irradiance) between $1 \mathrm{~mW}$ $300 \mathrm{~mW}$. LLLT, unlike other medical laser procedures, is not an ablative or thermal mechanism. Rather, it is a photochemical effect equivalent to photosynthesis in plants, whereby the light is absorbed and exerts a chemical change.' Low level NIR light penetrates more deeply than ultraviolet (UV) or visible light, and does not harm living tissue. ${ }^{2}$ NIR light has relatively high penetrability of biological tissues, and its light scattering is somewhat less than that of the visible light. Already applied in numerous areas, laser-induced phototherapy has been widely accepted as a universal treatment, and the reliance on the laser as a form of treatment is still growing. Interest in LLLT is increasing, especially during the past decade, and the number of related studies and clinical trials performed has markedly increased. Many animal models and clinical studies have established the therapeutic value of LLLT on a variety of diseases and injuries, and LLLT has been widely used in both chronic and acute conditions. ${ }^{1}$ LLLT has been discovered to be effective in wound healing, dental disease, chronic pain, musculoskeletal complications, enhancement of various biological processes, including neovascularization, and in the promotion of angiogenesis. LLLT increases cell proliferation, which positively modulates the processes of repair. Although the exact mechanisms of laser therapy is not fully clarified, it has been documented that once absorbed, the light can modulate cell biochemical reactions and stimulate a mitochondrial respiration, enhancing the production of molecular oxygen, ATP synthesis and collagen deposition. Overall, the prevailing opinion is that the respiratory chain plays a central role in the effect induced by laser therapy.

Gentamicin (GM) is one of the aminoglycosides that causes ototoxicity, but it is still widely used clinically because of its effectiveness and low cost. Gentamicin is known to cause vestibular and cochlear ototoxicity via destruction or damage to the structure and function of vestibular inner ear. The resulting damage from gentamicin can vary from very minimal to complete loss of vestibular function. ${ }^{3}$ Various molecules have been used to prevent and treat aminoglycoside-induced ototoxicity such as deferoxamine and MK-801. ${ }^{4}$ LLLT has been effective to prevent and recover cochlear hair cells after gentamicin ototoxicity in vitro study. ${ }^{5}$ LLLT has been effective to recue noise induced hearing loss in rats. ${ }^{6}$ However, no study in the literature has investigated the effect of LLLT on the recovery of vestibular dysfunction and damaged vestibular hair cells after ototoxicity. However, the effect of LLLT on neural cell recovery has been reported by several authors. ${ }^{7-9}$ Considering that neural cells and cochlear hair cells responded to LLLT, recovery of ototoxic vestibular dysfunction by trnasmeatal LLLT application to the vestibular inner ear may also be expected.

The purposes of this study were to measure a penetration rate of transmeatal LLLT into the inner ear through a tympanic membrane, and assess the preventive or therapeutic effects of transmeatal LLLT applied to the gentamicin-induced vestibular inner ear to recover from gentamicin-induced vestibulopathy, using earth vertical and off vertical axis rotation tests.

\section{MATERIALS AND METHODS}

\section{Animal model and experimental protocol}

Adult male guinea pigs, 12-week-old, supplied by Narabio Laboratories (Seoul, Korea) and weighing approximately 300-400 g were used for this study. All experimental procedures were performed in accordance with the guidelines of Animal Use Committee of the Dankook University College of Medicine. The animals were anesthetized before each gentamicin injection and transmeatal LLLT irradiation. Zoletil (Virbac Laboratories, France) and Rumpun (Bayer Korea, Korea) were mixed in a 4:1 ratio for anesthetics. The mixture was given by intramuscular injection, and boosted with one-fifth of the original dose as required. Approximately $0.1 \mathrm{ml}$ of the anesthetics was administered for every $100 \mathrm{~g}$ body weight.

\section{Transmeatal penetration of LLLT}

We have measured penetration rate of tranasmeatal LLL into the perilymphatic space of cochlea through an a tympanic membrane, and cochlea wall with a diode laser (EIT 21, Shinsung, Seoul Korea, Fig. 1) that has $830 \mathrm{~nm}$ wavelength with output power of $80 \mathrm{~mW}$. First, $80 \mathrm{~mW}$ was irradiated into the external ear canal opening and the penetration was measured at the middle ear cavity and perilymphatic space using two temporal bones from a human cadaver. In the next study, $80 \mathrm{~mW}$ was irradiated into the external ear canal of 5 guinea pigs for $30 \mathrm{~min}$ $\left(144 \mathrm{~J} / \mathrm{cm}^{2}\right)$ daily for 2 weeks to see any adverse effects of tranasmeatal LLLT on the ear structures and changes of 
hearing. Histopathological studies of external ear canal skin and ear drum were carried out and auditory brain stem response (ABR) was performed to test any hearing changes after LLLT.

\section{LLLT on vestibular inner ear}

Twenty guinea pigs were randomly divided into the control and LLLT group assigning 10 animals in each group. All animals in both groups received GM $2.4 \mathrm{mg}$ injected into the left middle ear cavity through a tympanic membrane once a day for two days. The LLLT group animals received transmeatal LLLT, $120.6 \mathrm{~J} / \mathrm{cm}^{2} 167 \mathrm{~mW} /$ $\mathrm{cm}^{2}$ for $30 \mathrm{~min}$ ) per day for 5 days using the EIT 21 diode laser (Fig. 1). Auricles were amputated for an effective delivery of LLL into ear canals. Only the left ear of LLLT

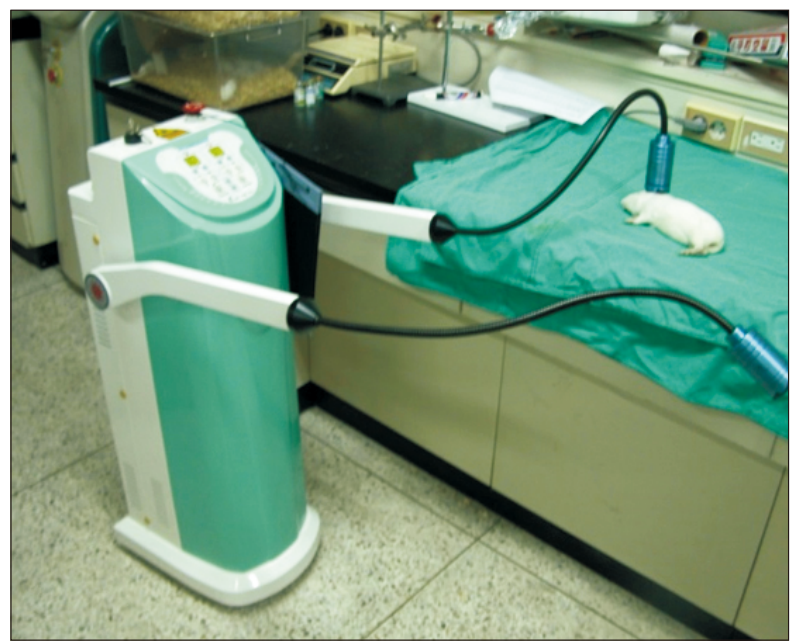

Fig. 1. Low level laser therapy into left ear using EIT 21 diode laser. Auricles were amputated for an effective delivery of low level laser into ear canals. group was given transmeatal LLLT.

\section{Slow Harmonic Acceleration (SHA) testing}

The animal rotator (Jeil Co. Seoul, Korea) is computer controlled system to rotate the animal and monitor eye movements. It records slow harmonic acceleration (SHA). All the animals were placed in prone position on mounting plate of animal rotator so that the horizontal semicircular canals are in the plane of rotation and firmly strained so that it follows chair motion (Fig. 2). Slow harmonic acceleration (SHA) using animal rotator was performed to 20 animals in both groups. SHA testing was performed at $0.16 \mathrm{~Hz}$ with peak angular velocity of 60 degrees/ second and at both counterclockwise and clockwise directions. The off vertical axis rotation counterclockwise and clockwise directions also were obtained separately. Statistical analysis was obtained using Wilcoxon signed ranks test (SPSS version 11.0). SHA tests have been performed on all 20 animals in both groups 1 day post completion of 5 days transmeatal LLLT into the left ear.

\section{RESULTS}

\section{Transmeatal penetration of LLL}

Transmeatal penetration of LLL was $4 \mathrm{~mW}(5 \%)$ at the

Table 1. Transmeatal penetration rate of low level laser therapy

\begin{tabular}{lcr}
\hline & Penetration through TM & Penetration through cochlea wall \\
\hline Cadaver & $4 \mathrm{~mW}(5 \%)$ & $1.6 \mathrm{~mW}(2 \%)$ \\
Guinea Pig & $6.4 \mathrm{~mW}(8 \%)$ & $4 \mathrm{~m}(4 \%)$ \\
\hline
\end{tabular}

No penetration through mastoid bone.

Histopathology of external ear canal skin and drum: normal.

$\mathrm{TM}$, tympanic membrane.
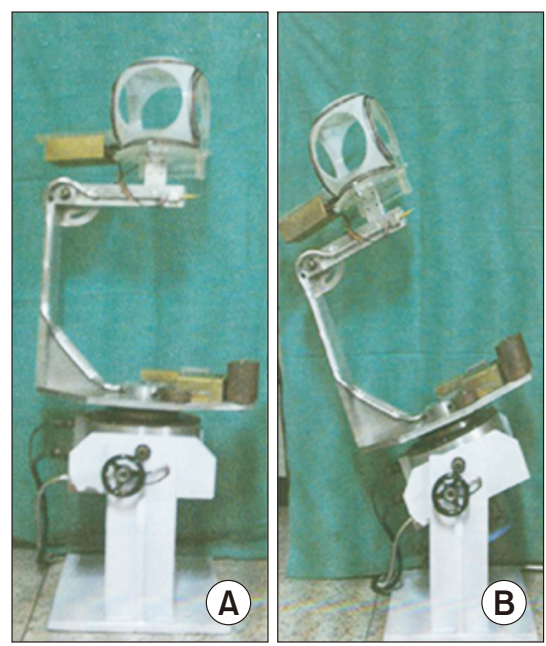

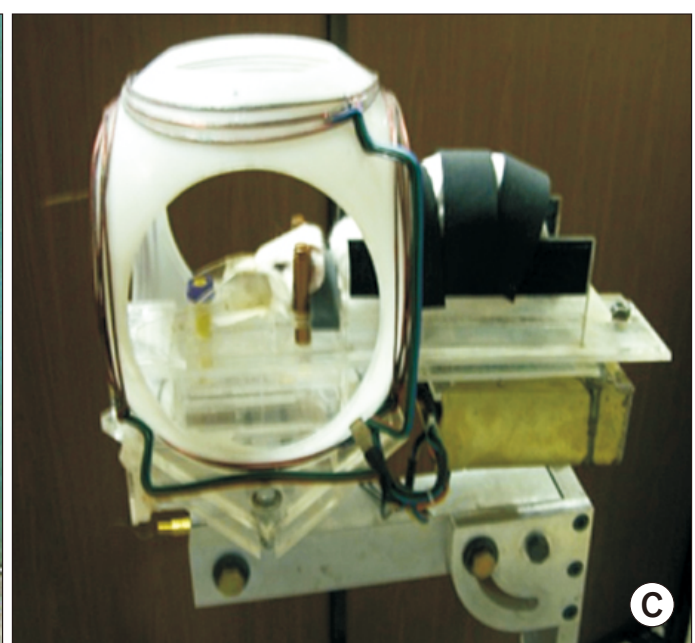

Fig. 2. Animal rotator. (A) Vertical axis position, (B) Off vertical position, (C) Guinea pig in testing position. 

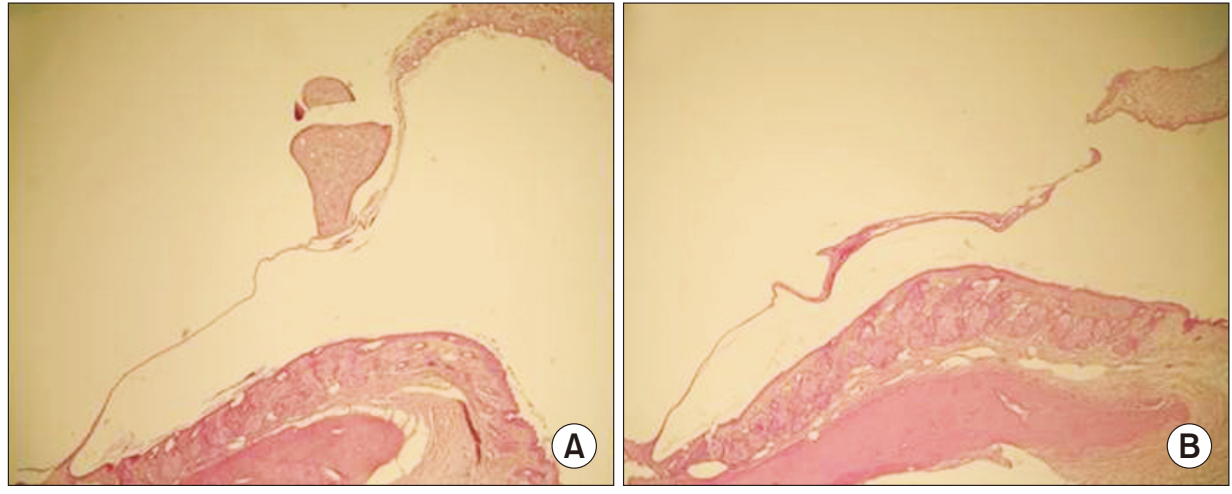

Fig. 3. Histopathology of tympanic membrane of unirradiated and irradiated ears. Tympanic membranes of both groups were intact and normal. External ear canal skins were also intact and normal without any signs of inflammation. (A) Unirradiated ear, (B) Irradiated ear.

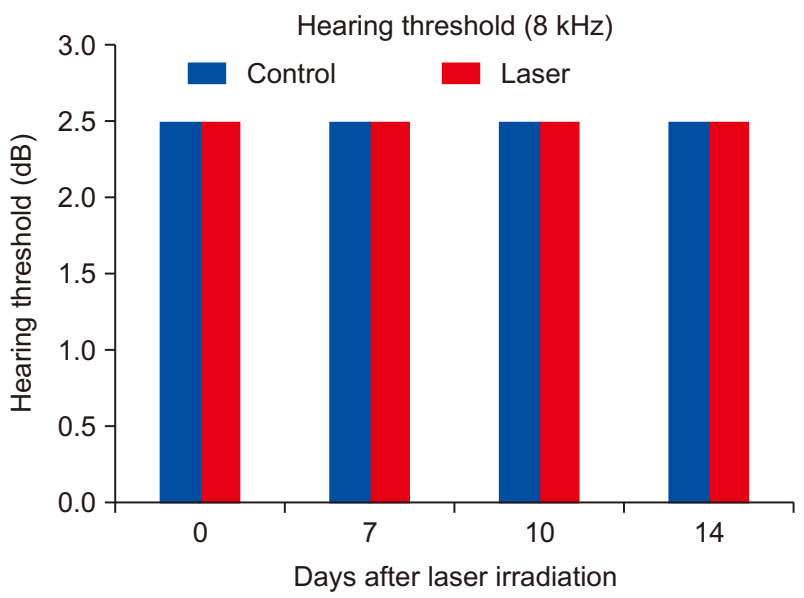

Fig. 4. Auditory brain response hearing tests revealed no hearing loss after low level laser therapy for 2 weeks.

middle ear cavity and $1.6 \mathrm{~mW}(2 \%)$ at the perilympnatic space in human cadaver temporal bones. Those values in guinea pigs were $6.4 \mathrm{~mW}(8 \%)$ at the middle ear space behind the tympanic membrane and $4 \mathrm{~mW}(5 \%)$ at the perilymphatic space. No penetration into perilymphatic space was measurable through the mastoid bone (Table 1).

\section{Histopathology of external canal and tympanic membrane and hearing test}

Histopathology studies of the ear canal skin and tympanic membrane showed all normal findings (Fig. 3). ABR hearing tests revealed no hearing loss after LLLT for 2 weeks (Fig. 4).

\section{Effects of transmeatal LLLT on vestibular inner ear (SHA Testing)}

The gain and modulation values from the animals were compared between the control and LLLT groups. Only left ear has been exposed to GM. The values from left
Table 2. Changes of the gain in Slow Harmonic Acceleration test, testing horizontal semicircular canal function

\begin{tabular}{lcc}
\hline & Left ear (GM) & Right ear \\
\hline No laser control group & $0.55^{*}$ & 0.80 \\
LLLT group & 0.71 & 0.76 \\
\hline
\end{tabular}

${ }^{*} p<0.05$.

GM, gentamicin; LLLT, low level laser therapy.

Table 3. Change of the modulation in off vertical axis rotation test (Otolithic Organs)

\begin{tabular}{lcc}
\hline & Left ear (GM) & Right ear \\
\hline No laser control group & $0.57^{*}$ & 0.65 \\
LLLT group & 0.75 & 0.64 \\
\hline
\end{tabular}

$* p<0.05$.

GM, gentamicin; LLLT, low level laser therapy.

directing counterclockwise rotation represents left ear function and that from right directing clockwise rotation represents right ear function. The gain of SHA test from counterclockwise rotation of the gentamicin injected control group was 0.55 that was significantly decreased comparing to 0.71 of LLLT group ( $p<0.05$ )(Table 2). On clockwise rotation, the gain of control and LLLT groups were 0.76 and 0.80 that were not significantly different. On off vertical axis rotation testing, the modulation value of counterclockwise rotation of the control group was 0.57 that was a significant decrease compared to 0.75 of the LLLT group. On clockwise rotation, the values were 0.65 in control group and 0.64 in laser group that were not a significant change $(p<0.05)$ (Table 3$)$.

\section{DISCUSSION}

The penetration study was performed to see how 
much LLL can penetrate through an ear drum and cochlear bony wall and reach into the inner ear. The study demonstrated that $5 \%$ of the LLLT was penetrated through the ear drum and $2 \%$ was done through the ear drum and cochlea bony wall into the perilymphatic space in human temporal bone. And $8 \%$ of the LLL was penetrated through the ear drum and $5 \%$ was passed through the ear drum and cochlea bony wall into the perilymphatic space in guinea pig temporal bone. This indicates that transmeatal LLL can reach into an inner ear and should be able to influence various functons of inner ear nerve cells. This study also showed that LLL does not penetrate through mastoid cavity to reach in an inner ear space. Transmastoid LLLT is no longer in use. Histopathologic studies performed after transmeatal LLLT demonstrated no pathologic changes of external canal skin and tympanic membrane. ABR taken after LLLT showed no hearing loss. This indicates that transmeatal irradiation of $144 \mathrm{~J} / \mathrm{cm}^{2}$ of LLL for 2 weeks into guinea pig ear canal does not cause any side effects in the external ear canal, tympanic ear drum, and inner ear including hearing. This is the first study to demonstrated that transmeatal LLLT does not cause any adverse effect to the canal skin, tympanic ear drum, and cochlea.

In our second study, we intended to see a preventive and/or therapeutic effect of LLLT on otolithic organ and horizontal semicircular canal ampulla after GM injection into middle ear of guinea pigs. Part of GM in the middle ear is to penetrate through a round window membrane into perilymphatic space, inducing ototoxic effects on cochlea and vestibular inner ear. This study demonstrated the preventive and/or therapeutic effect of $L L L$ by preventing reduction of gain and modulation in laser group animals while they were reduced significantly in control group animals. We expect that LLL has penetrated through the ear drum, labyrinthine bony wall and oval window membrane and it was able to improve vestibular dysfunction of the saccule, utricle, and ampulla of semicircular canals. There have been many studies of LLLT on the cochlea and tinnitus, but none on vestibular inner ear could be found. The LLL used in this study had wavelength of $830 \mathrm{~nm}$ that is capable to penetrate better than 600-700 $\mathrm{nm}$ wavelength LLL that was used in many previous studies. The fluence of $144 \mathrm{~J} / \mathrm{cm}^{2}$ that was used in this study is higher than any dosage used in previous animal and clinical studies. Since only $2 \%$ of the transcanal LLL can penetrated into the inner ear, it would be necessary to find out a maximum power of LLL that can be irradiated via external ear canal without inducing any side effects in middle and inner ear structures and their functions.

Although the exact mechanisms of laser therapy have not been clarified, presently the use of LLLT to the left ears of the rats with gentamicin ototoxicity significantly improved the vestibular dysfunction, compared to the vestibular function of the control group right ear. Transmeatal LLLT into left ear improved vestibular function, and could have decreased the amount of apoptosis of ampular hair cells, that ultimately positively modulated the repair processes. It was demonstrated that transmeatal infrared laser therapy applied 6 hours post-embolic stroke can improve the clinical rating scores in rabbits. ${ }^{10}$ The temporal improvement in the neurological functional performance by LLLT may also suggest a positive effect on neurogenesis. These effects can be attributed to a photochemical mechanism that is based on light absorption by mitochondrial chromophores. Thus, the rescue effect of LLLT observed in this study is considered to have resulted from these various mechanisms. From our earlier studies, daily LLLT with a laser diode $632 \mathrm{~nm}$ for 30 days to the gentamicin insulted utricular explants demonstrated regeneration and proliferation of utricular hair cells. ${ }^{11}$ Loss of hair cells was nearly stopped 2 days after exposure to gentamicin; a peak of regeneration was reached after 18 days of LLLT and sustained for 2 weeks in the medium with the continuous LLLT. In that study of preventive and therapeutic LLLT on gentamicin insulted utricular explants was demonstrated. It has been demonstrated that LLLT before and after GM insult on utricular hair cells was very effective to prevent and treat gentamicin ototoxicity. ${ }^{11}$ The results of this previous study suggested that LLLT promotes hair cell regeneration following gentamicin damage in utricular explants. Since LLLT was effective in vitro study, it was expected to be effective in vivo study as shown in this study. LLLT has been shown to be effective in the treatment of gentamicininduced ototoxicity. ${ }^{5}$ The presently demonstrated positive results on vestibular ototoxicity may provide further clinical application of LLLT. Further studies are required to demonstrate the efficacy of delayed treatments on chronic vestibulopathy and for detailed understandings of its mechanisms.

\section{CONCLUSION}

The present study demonstrated that transmeatal LLLT does not induce any side effects to the ear canal skin, ear drum and hearing. The transmeatal LLLT also restored the decreased vestibular dysfunction induced by GM 
ototoxicity in guinea pigs. Transmeatal LLLT may have clinical implications in the treatment of various vestibular diseases. Further studies are essential to verify the exact mechanisms and the most effective application of LLLT to rescue vestibular dysfunction.

\section{ACKNOWLEDGMENTS}

This study was supported by Medical Laser Research Fund from MOTIE (Ministry of Technology, Industry, and Energy), and KIAT.

\section{REFERENCES}

1. Huang YY, Chen AC, Carroll JD, Hamblin MR. Biphasic dose response in low level light therapy. Dose Response 2009;7:35883.

2. Wong-Riley MT, Liang HL, Eells JT, Chance B, Henry MM, Buchmann E, et al. Photobiomodulation directly benefits primary neurons functionally inactivated by toxins: role of cytochrome c oxidase. J Biol Chem 2005;280:4761-71.

3. Basile AS, Brichta AM, Harris BD, Morse D, Coling D, Skolnick P. Dizocilpine attenuates streptomycin-induced vestibulotoxicity in rats. Neurosci Lett 1999;265:71-4.

4. Roland JT, Cohen NL. Vestibular and auditory ototoxicity. In: Cummings CW, Frederixkson JM, Harker LA, Krause CJ, Schuller DE, Richardson MA, editors. Otolaryngology and Head and Neck Surgery. 3rd ed. St Louis: Mosby Year Book; 1998. p.3186-97.

5. Rhee CK, He P, Jung JY, Ahn JC, Chung PS, Suh MW. Effect of low-level laser therapy on cochlear hair cell recovery after gentamicin-induced ototoxicity. Lasers Med Sci 2012;27:98792.

6. Rhee CK, Bahk CW, Kim SH, Ahn JC, Jung JY, Chung PS, et al. Effect of low-level laser treatment on cochlea hair-cell recovery after acute acoustic trauma. J Biomed Opt 2012;17:068002.

7. Detaboada L, Ilic S, Leichliter-Martha S, Oron U, Oron A, Streeter J. Transcranial application of low-energy laser irradiation improves neurological deficits in rats following acute stroke. Lasers Surg Med 2006;38:70-3.

8. Oron A, Oron U, Chen J, Eilam A, Zhang C, Sadeh M, et al. Lowlevel laser therapy applied transcranially to rats after induction of stroke significantly reduces long-term neurological deficits. Stroke 2006;37:2620-4.

9. Zivin JA, Albers GW, Bornstein N, Chippendale T, Dahlof B, Devlin T, et al; NeuroThera Effectiveness and Safety Trial-2 Investigators. Effectiveness and safety of transcranial laser therapy for acute ischemic stroke. Stroke 2009;40:1359-64.

10. Lapchak PA, Wei J, Zivin JA. Transcranial infrared laser therapy improves clinical rating scores after embolic strokes in rabbits. Stroke 2004;35:1985-8.

11. Chung YW, Ahn JC, Lim ES, Kim YS, Lee SH, Lee MY, et al. A promotive effect of low-level laser on hair cell regeneration following gentamicin induced ototoxicity in postnatal organotypic culture of rat utricles. Korean J Otolaryngol-Head Neck Surg 2007;50:25-30. 